\title{
Die Urteilspublikation gehört ins E-Justice-Gesetz
}

Obwohl die elektronische Urteilspublikation ein zentrales Element von E-Justice ist, fehlt im Entwurf für ein Bundesgesetz über die Plattform für die elektronische Kommunikation in der Justiz eine Regelung dazu. Nachdem eine im ZPO-Vorentwurf enthaltene Bestimmung mit dem Argument verworfen wurde, dass die Frage nicht nur das Zivilverfahren betreffe, erscheint es naheliegend, die Thematik im E-Justice-Gesetz aufzunehmen. Die AutorInnen des vorliegenden Beitrags formulieren einen konkreten Vorschlag.

Beitragsart: Forum 


\section{Inhaltsübersicht}

I. Einleitung

II. Gründe für die Publikation von Urteilen

III. Publikation als zentrales Element von E-Justice

IV. Welche Regelungen sind nötig?

A. Formate und Metadaten

B. Publikationspflicht

C. Formulierungsvorschlag

V. Ausblick

\section{Einleitung}

[1] Am 11. November 2020 hat der Bundesrat den Vorentwurf zu einem Bundesgesetz über die Plattform für die elektronische Kommunikation in der Justiz (BEKJ) in die Vernehmlassung geschickt ${ }^{1}$. Während der Vorentwurf keinen Kurztitel enthält, wird das Gesetz in den Jahreszielen des Bundesrates 2020 als E-Justice-Gesetz bezeichnet ${ }^{2}$. Das Gesetz bezweckt gemäss Art. 1 Abs. 1 des Vorentwurfs ${ }^{3}$ «die Gewährleistung einer sicheren und einfachen elektronischen Kommunikation in der Justiz zwischen Privaten und Behörden sowie unter Behörden». Es regelt gemäss Art. 1 Abs. 2 des Vorentwurfs den Aufbau und Betrieb einer zentralen Plattform für die Übermittlung von elektronischen Dokumenten in der Justiz (E-Justiz-Plattform), die Schaffung einer öffentlichrechtlichen Körperschaft als Trägerschaft der Plattform sowie allgemeine verfahrensrechtliche Aspekte der elektronischen Kommunikation und Akteneinsicht.

[2] Was im Vorentwurf jedoch fehlt, sind Bestimmungen zur elektronischen Publikation von Urteilen. Es bietet sich aus verschiedenen Gründen an, diese Thematik im E-Justice-Gesetz aufzunehmen.

\section{Gründe für die Publikation von Urteilen}

[3] Über die Gründe für die Publikation von Urteilen wurde schon viel geschrieben ${ }^{4}$, deshalb sollen sie hier nur kurz zusammengefasst werden:

[4] Sowohl die Bundesverfassung als auch die EMRK und der UNO-Pakt II enthalten eine Pflicht zur öffentlichen Urteilsverkündung ${ }^{5}$. Schutzzwecke der Urteilsöffentlichkeit sind die Unterbindung von Geheimjustiz, die Transparenz der Justiztätigkeit und die Schaffung von Vertrauen in den Rechtsstaat. Das Bundesgericht hat schon vor zwanzig Jahren festgehalten, dass dem Zweck des Verkündungsgebots mit einer Publikation in Periodika oder auf dem Internet besser gedient

1 Medienmitteilung des Bundesrates vom 11. November 2020: Bundesrat schlägt zentrale Plattform für den elektronischen Rechtsverkehr vor.

2 Bundeskanzlei, Ziele des Bundesrates 2020, Band I, S. 12.

3 Vorentwurf vom 11. November 2020 für ein Bundesgesetz über die Plattform für die elektronische Kommunikation in der Justiz.

4 Oskar Bosshardt, Bemerkungen zur Veröffentlichung von Entscheiden, ZBl 1966, S. 345 ff.; Daniel HürLimanN, Verbesserte Zugänglichkeit zu Gerichtsurteilen, NZZ vom 31. August 2016, S. 9; Romain JordAn, Accès à la jurisprudence: le TF impose la transparence, plaidoyer 5/17, S. 32 ff.; SABine Steiger-SAckmann, Transparentere Justiz, sui-generis 2019, S. 122; PAul TschüMPERLIN, Die Publikation gerichtlicher Entscheide, in: Daniel Kettiger / Thomas Sägesser (Hrsg.), Kommentar zum Publikationsgesetz des Bundes, Bern 2011, S. 69 ff.

5 Art. 30 Abs. 3 BV, Art. 6 Abs. 1 EMRK, Art. 14 Ziff. 1 UNO-Pakt II. 
sein dürfte als mit einer mündlichen Eröffnung des Urteils an einer Gerichtsverhandlung, «da das Publikum faktisch nur begrenzte Möglichkeiten hat, an derartigen Verhandlungen teilzunehmen» ${ }^{6}$.

[5] Rechtskonformes Verhalten ist nur möglich, wenn das Recht bekannt ist. Aus diesem Grund besteht eine staatliche Pflicht zur Publikation normativer Akte. Viele Rechtsfragen lassen sich jedoch nur unter Berücksichtigung der Rechtsprechung beantworten. Auch wenn keine Pflicht zur Publikation sämtlicher Urteile besteht, sprechen die Gründe, welche zur Publikationspflicht normativer Akte führen - namentlich das Rechtsstaatsprinzip und die Pflicht zur Wahrung von Treu und Glauben - auch für die vermehrte Publikation von Rechtsprechung.

\section{Publikation als zentrales Element von E-Justice}

[6] Der Verein eJustice.CH hat im Jahr 2016 eine Vision für E-Justice in der Schweiz erarbeitet und veröffentlicht ${ }^{7}$. Die Vision enthält den folgenden Leitsatz: «Justizverfahren werden in elektronischer Form anhängig gemacht, geführt, abgeschlossen und publiziert. Sämtliche Verfahrensschritte und sämtlicher Geschäftsverkehr laufen elektronisch ab.» Bereits aus diesem Satz ergibt sich, dass die Publikation zentraler Bestandteil von E-Justice ist. Die Vision enthält sodann Konkretisierungen mit Blick auf Behörden, NutzerInnen und die Gesellschaft. Im Abschnitt betreffend die Gesellschaft findet sich der folgende Absatz:

«Erfolgt der gesamte Prozessablauf wie oben dargelegt in elektronischer Form und liegen somit die erforderlichen Daten bereits in elektronischer Form vor, kann eine umfassende elektronische Publikation von Entscheiden in anonymisierter Form ohne hohen personellen, technischen oder finanziellen Aufwand erreicht werden. Mit einer umfassenden Publikation der Entscheide wird nicht nur die Transparenz der Justiz verbessert. Der Wissenschaft werden auch die Datengrundlagen bereitgestellt, um die Justizpraxis wissenschaftlich zu begleiten und zu einer Verbesserung zum Nutzen aller beizutragen.»

[7] Im Rahmen der laufenden ZPO-Revision hat der Bundesrat vorgeschlagen, dass er für Entscheide, die elektronisch publiziert werden, Regelungen erlassen kann, insbesondere über die Zugänglichkeit der Entscheide sowie die zu verwendenden Formate und Metadaten ${ }^{8}$. Im Vernehmlassungsverfahren wurde dieser Vorschlag kritisiert und die Bestimmung des Vorentwurfs wurde in der Folge nicht in den Entwurf übernommen. Dem Bericht über die Ergebnisse des Vernehmlassungsverfahrens lässt sich entnehmen, dass die Bestimmung unter anderem deshalb kritisiert wurde, weil «die Frage auch noch andere Gebiete als das Zivilverfahren [betrifft], weshalb eine isolierte Bestimmung in der ZPO nicht gerechtfertigt ist» ${ }^{9}$. Sodann wurde vorgeschla-

Urteil des Bundesgerichts 1P.229/2001 vom 2. Oktober 2001, E. 2c.

7 Verein eJustice.CH, Eine Vision für eJustice in der Schweiz, November 2016 (abrufbar unter www.ejustice.ch Projekte Vision eJustice).

8 Art. 400 Abs. $2^{\text {bis }}$ des ZPO-Vorentwurfs vom 2. März 2018.

9 Bundesamt für Justiz, Übersicht über das Ergebnis des Vernehmlassungsverfahrens zur Revision der Zivilprozessordnung vom 29. Januar 2020, S. 58. 
gen, ein Spezialgesetz zu schaffen, das alle Rechtsgebiete abdeckt ${ }^{10}$. Mit dem Vorentwurf für ein E-Justice-Gesetz liegt nun ein solches Spezialgesetz vor und es drängt sich mit Blick auf die Ergebnisse des Vernehmlassungsverfahrens zur ZPO-Revision geradezu auf, das Thema in diesem Gesetz aufzunehmen.

\section{Welche Regelungen sind nötig?}

[8] Eine Regelung betreffend Urteilspublikation sollte einerseits die bereits im ZPO-Vorentwurf vorgeschlagene Vereinheitlichung von Formaten und Metadaten sowie andererseits eine Verpflichtung zur Publikation aller zweitinstanzlichen sowie (mindestens) einer Auswahl von erstinstanzlichen Urteilen umfassen.

\section{A. Formate und Metadaten}

[9] Die Gründe für den Erlass von Vorgaben betreffend Formate und Metadaten hat der Bundesrat bereits im erläuternden Bericht zum ZPO-Vorentwurf genannt. Die entsprechende Passage wird nachfolgend im Originalwortlaut wiedergegeben ${ }^{11}$ :

«Nach Artikel 54 Absatz 1 ZPO sind Entscheide der Öffentlichkeit zugänglich zu machen; dies dient der (demokratischen) Kontrolle der Justiz durch die Öffentlichkeit und der Schaffung von Transparenz. Heute wird diese Öffentlichkeit häufig durch die elektronische Publikation im Internet hergestellt, was mit erheblichen Vorteilen und Ersparnissen verbunden ist und die Zugänglichkeit der Entscheide deutlich erleichtert. Diese Vorteile werden in der Praxis teilweise wieder aufgehoben oder zumindest gefährdet: Heute zeigt sich schweizweit eine Vielfalt unterschiedlicher Formen der elektronischen Entscheidpublikation. So werden unterschiedliche Dateiformate verwendet, teilweise Gesamtdateien für die Leitentscheide ganzer Jahrgänge publiziert, oder die Entscheide werden in besonderen Datenbanken publiziert. Diese Unterschiede erschweren insbesondere die Auffindbarkeit und die Suchbarkeit in den Entscheiden, was sich darin zeigt, dass die Entscheide oftmals mit gängigen Suchmaschinen wie 〈google〉 nicht gefunden oder falsche Treffer produziert werden. Es ist aber gerade im öffentlichen Interesse, dass die Zugänglichkeit elektronisch publizierter Entscheide möglichst uneingeschränkt ist und keine unnötigen Zugangsschranken bestehen. Diese lassen sich ohne nennenswerten Aufwand beseitigen. Dazu sind aber schweizweit einheitliche Regelungen und Vorgaben über die Zugänglichkeit der Entscheide sowie die zu verwendenden Formate und Metadaten notwendig. Ob eine solche Kompetenz im geltenden Artikel 400 Absatz 1 ZPO, der den Bundesrat zum Erlass von Ausführungsbestimmungen ermächtigt, enthalten ist, ist unklar. Der Bundesrat soll daher in einem neuen Absatz $2^{\text {bis }}$ von Artikel 400 ZPO, der die Grundsätze des Voll-

10 Ebenda, S. 58

11 Bundesrat, Erläuternder Bericht zur Änderung der Zivilprozessordnung (Verbesserung der Praxistauglichkeit und der Rechtsdurchsetzung) vom 2. März 2018, S. 95. 
Daniel Hürlimann / Sébastien Fanti / Christian Laux / Adrian Rufener / Claudia Schreiber, Die Urteilspublikation gehört ins E-Justice-Gesetz, in: «Justice - Justiz - Giustizia» 2020/4

zugs der ZPO regelt, zukünftig ausdrücklich ermächtigt werden, in diesem Bereich entsprechende Ausführungsbestimmungen zu erlassen.»

[10] Dieser Begründung ist nichts hinzuzufügen. Weil sich die im ZPO-Entwurf vorgeschlagene Bestimmung jedoch auf die Regelung von Formaten und Metadaten beschränkt hat, wird sie beim nachfolgenden Formulierungsvorschlag nicht unverändert übernommen.

\section{B. Publikationspflicht}

[11] Weil trotz der genannten Gründe für die Publikation von Urteilen häufig nur eine sehr beschränkte und teilweise von sachfremden Kriterien beeinflusste Auswahl von Urteilen veröffentlicht wird, ist in einem geeigneten Gesetz auch eine Publikationspflicht zu statuieren. Dabei ist abzuwägen, ob die Publikationspflicht für sämtliche Urteile aller Instanzen eingeführt werden soll oder ob eine eingeschränkte Publikationspflicht vorzuziehen ist.

[12] Für eine umfassende Publikationspflicht sprechen die Schwierigkeit einer Bewertung von Urteilen im Hinblick auf ihre Publikationswürdigkeit ${ }^{12}$ und die Gefahr, dass bestimmte Urteile weiterhin aus sachfremden Kriterien nicht publiziert werden. Gegen eine umfassende Publikationspflicht spricht allenfalls der damit einhergehende Aufwand. Dieser entsteht in erster Linie bei der Anonymisierung, lässt sich allerdings mit verschiedenen Massnahmen stark reduzieren. Der Aufwand für die Anonymisierung kann erstens durch anonymisierungsfreundliches Verfassen von Urteilen, zweitens durch technologische Unterstützung und drittens durch den Erlass von klaren Vorgaben zur Tiefe der Anonymisierung reduziert werden ${ }^{13}$. Betreffend technologische Unterstützung und Erlass von Vorgaben lohnt sich ein Blick auf Frankreich. Die dortige Gesetzgebung legt fest, dass im Regelfall lediglich der Vor- und Nachname unkenntlich gemacht werden. Eine darüber hinausgehende Anonymisierung ist nur dann vorzunehmen, wenn andernfalls die Sicherheit oder die Privatsphäre der betroffenen Personen beeinträchtigt würden ${ }^{14}$. Zudem wird im Rahmen eines Pilotprojekts am höchsten Gericht Frankreichs (Cour de Cassation) das Potential der künstlichen Intelligenz für die Anonymisierung von Urteilen untersucht ${ }^{15}$.

[13] Trotz dieser Möglichkeiten zur Reduktion des Anonymisierungsaufwands ist davon auszugehen, dass dieser insbesondere bei erstinstanzlichen Urteilen auch in Zukunft nicht zu vernachlässigen ist. Dementsprechend erscheint uns eine umfassende Publikationspflicht aus heutiger Sicht nicht zwingend. Aus Sicht der Anwaltschaft wäre schon viel gewonnen, wenn alle zweitinstanzlichen Urteile publiziert würden. Eine Pflicht zur Publikation sämtlicher zweitinstanzlicher Entscheide wurde auch im Rahmen der Vernehmlassung zum ZPO-Vorentwurf gefordert ${ }^{16}$. Eine solche Pflicht entspricht somit einem allgemeinen Bedürfnis und ist mit Blick auf Rückmeldungen in einem bereits durchgeführten Vernehmlassungsverfahren auch legitimiert. Für erstin-

12 Daniel Hürlimann, Publikation von Urteilen durch Gerichte, sui-generis 2014, S. 82 ff., Rn. 40.

Als Beispiel kann auf die Regeln des Bundesgerichts für die Anonymisierung der Urteile (Grundsätze gemäss Beschluss der Präsidentenkonferenz und der Verwaltungskommission) verwiesen werden.

14 Article L111-13 Abs. 2 Code de l'organisation judiciaire; Article L10 Abs. 3 Code de justice administrative.

15 Cour de Cassation, Deux spécialistes de l'intelligence artificielle travaillent à la Cour de cassation sur la méthode d'anonymisation des décisions de justice.

16 Bundesamt für Justiz, Übersicht über das Ergebnis des Vernehmlassungsverfahrens zur Revision der Zivilprozessordnung vom 29. Januar 2020, S. 58: «Es wird verlangt, eine Pflicht zur Publikation sämtlicher zweitinstanzlicher Entscheide vorzusehen». 
stanzliche Urteile kann man sich auf eine Auswahl beschränken. Diese sollte sich an sachlichen Kriterien orientieren. Der Anwendung sachfremder Kriterien bei der Auswahl der zu publizierenden erstinstanzlichen Urteile könnte mit einem unabhängigen Auswahlgremium vorgebeugt werden.

\section{Formulierungsvorschlag}

[14] Das E-Justice-Gesetz ist gemäss Art. 2 des Vorentwurfs auf Verfahren anwendbar, soweit das jeweilige Verfahrensrecht dies vorsieht. Aus diesem Grund eignet es sich nicht, um eine Publikationspflicht zu statuieren. Hingegen ist das E-Justice-Gesetz das ideale Gefäss, um die technischen und rechtlichen Voraussetzungen für eine zentrale Urteilspublikation zu schaffen.

[15] Nach den Allgemeinen Bestimmungen und einem Abschnitt zur Trägerschaft der Plattform sind im dritten Abschnitt des Vorentwurfs für ein E-Justice-Gesetz die Funktionen der Plattform geregelt.

[16] Eine weitere Funktion soll die Entscheidpublikation sein. In diesem Abschnitt könnte dementsprechend ein neuer Artikel mit dem folgenden Wortlaut aufgenommen werden:

\section{Art. X Entscheidpublikation}

${ }^{1}$ Die E-Justiz-Plattform stellt für Gerichte und Behörden ein Modul für die Publikation von Entscheiden zur Verfügung.

2 Die über dieses Modul publizierten Entscheide sind frei zugänglich (Zugang ohne Authentifizierung).

${ }^{3}$ Der Bundesrat regelt die zu verwendenden Formate und Metadaten.

\section{Ausblick}

[17] Mit der vorgeschlagenen Regelung würde der Zugang zum Recht für alle verbessert. Für Gerichte und Behörden würde der Aufwand für die Publikation von Entscheiden abnehmen, da sie direkt auf der ohnehin verwendeten Plattform erfolgt. Für RechtsanwältInnen, für die Wissenschaft, für alle anderen am Recht Interessierten und auch für die Gerichte und Behörden selbst würde die Plattform die schweizweite Recherche nach Rechtsprechung deutlich erleichtern. Gleichzeitig ist davon auszugehen, dass die Kosten für ein Publikationsmodul nicht nur überschaubar, sondern deutlich tiefer wären als die heute anfallenden Kosten für den parallelen Betrieb von 90 Publikationslösungen ${ }^{17}$.

Daniel HÜrlimann ist Assistenzprofessor für Informationsrecht an der Universität St. Gallen, Rechtsanwalt bei Laux Lawyers sowie Vorstandsmitglied der Vereine eJustice.ch und entscheidsuche.ch.

17 Peter Guyan, Zugänglichkeit von schweizerischen Gerichtsentscheiden im Internet, in: «Justice - Justiz - Giustizia» 2018/2, Rz. 10. 
Daniel Hürlimann / Sébastien Fanti / Christian Laux / Adrian Rufener / Claudia Schreiber, Die Urteilspublikation gehört ins E-Justice-Gesetz, in: «Justice - Justiz - Giustizia» 2020/4

SÉBASTIEn FANTI ist Datenschutzbeauftragter des Kantons Wallis und mit seiner Kanzlei Teil des Kanzleinetzwerks Lexing.

Christian Laux ist Rechtsanwalt und Partner bei Laux Lawyers sowie Vizepräsident der Swiss Data Alliance.

Adrian Rufener ist Rechtsanwalt bei Amparo Anwälte und Notare sowie Richter am Verwaltungsgerichtshof des Fürstentums Liechtenstein.

Claudia Schreiber ist Inhaberin der Advokatur Schreiber sowie Vorstandsmitglied des Vereins entscheidsuche.ch und leitet den Support elektronische Kanzlei (SEK, eine Dienstleistung des Verbands bernischer Notare VbN und des Bernischen Anwaltsverbandes BAV). 